Sädhanā, Vol. 26, Part 5, October 2001, pp. 387-412. (C) Printed in India

\title{
Fundamentally excited flow past a surface-mounted rib. Part I: Turbulent structure characterisation
}

\author{
P K PANIGRAHI
}

Department of Mechanical Engineering, Indian Institute of Technology, Kanpur 208 016, India

e-mail: panig@iitk.ac.in

MS received 29 December 1999; revised 19 February 2001

\begin{abstract}
Different data analysis techniques for characterisation of the turbulent flow past a surface-mounted rib are reviewed. Deficiencies of the existing techniques are explained and modified techniques for determination of coherent structure magnitude and phase jitter are suggested. The effect of fundamental excitation on the flow is studied by using these turbulent signal analysis techniques. The appropriate length scale for characterizing the large-scale structures present in the reattaching shear layer of the surface-mounted rib is found to be the momentum thickness at the downstream edge of the rib, and the corresponding Strouhal number is 0.013 . This is in contrast to a rib in the free stream, where the rib height is the correct scaling parameter. The post reattachment region is observed to be dominated by large-scale structures contrary to the traditional belief that large eddies break into small scales at the reattachment location. Low magnitude of phase jitter in the near field region is observed, indicating coherence of the flow structures. Phase decorrelation begins to occur beyond three rib heights from the downstream edge of the rib. From the quadrant analysis results, the outer edge of the shear layer is observed to be dominated by large-scale ejection motions.
\end{abstract}

Keywords. Phase jitter; pattern recognition method; quadrant analysis; turbulator; coherent structures.

\section{Introduction}

Large scale structures play an important role in the development of free shear layers and jets and there is a large body of literature, experimental and analytical, dealing with this subject (see Hussain \& Zaman 1980, Ho \& Huere 1984, Yang \& Karlsson 1991, Rajaee \& Karlsson 1992, Zhou et al 1996). Ribbed duct flows are encountered in a variety of practical applications, for example, turbine blade cooling and heat exchangers, where it is desired to enhance the mixing in the separated shear layer behind the rib. The separated shear layer past the rib has been shown (Panigrahi \& Acharya 1996) to

A list of symbols is given at the end of the paper 
be dominated by coherent structures. It is expected that the flow and heat transport mechanisms would be largely influenced by the dynamics of these structures. Thus, there is a need for identifying the mechanisms associated with the production and transport of coherent turbulent structures.

For meaningful interpretation of turbulent data, different analysis techniques have been used. The primary goal of the data analysis techniques is to identify the individual modes present and to determine the evolution of the amplitudes and phases of these modes. But, in many cases the analysis is made more difficult by the jitter in the data or by the superimposed small-scale fluctuations. To enhance coherence, and to facilitate data analysis, many investigators have used a low-amplitude forcing signal to manipulate the flow. The most common data analysis approach has been to use phase-locked conditional sampling technique with the driving signal as a timing reference. Yang \& Karlsson (1991), Rajaee \& Karlsson (1992) and Hussain (1980) have used this approach to determine the large-scale structure amplitude. One limitation of the above approach is that it is accurate only if the phases of the structures of interest retain their coherence throughout the domain. But in reality, there will be significant jitter during the various stages of the evolution of the large scales, i.e. the formation, the growth, the re-orientation, the mutual interaction, and the breakdown of the large-scale structures. Rajaee \& Karlsson (1992) have further observed that identifying a single mode, for example the fundamental, is inadequate because of the involvement of other instability waves with different frequencies in the turbulent flow field. They considered four instability modes, the fundamental, its first sub-harmonic, 3/2 fundamental and the first harmonic to study the evolution of large-scale structures. However, they observed that the approach is not successful beyond the first vortex pairing stage where the flow becomes more chaotic and there is considerable phase jitter. Hence, any real practical definition of coherent motion should consider not only all the instability modes but also the relative phase jitter among themselves.

Zhou et al (1996) have proposed a pattern-recognition approach to quantify coherent structures and have used it to study the coherent and random motion in a forced plane wall jet. They observed that phase-locked and ensemble-averaged procedures do not correctly represent the energy contained in the coherent structure. Their pattern recognition approach appeared to provide the correct partitioning of energy between the several different modes selected to represent the coherent structures. However, they proposed further testing of their technique in a variety of applications. The present work aims to examine the pattern recognition technique for characterizing the large-scale structures present in the reattaching shear layer of a surface-mounted rib.

To determine the optimum forcing criteria for manipulating the flow behind a surfacemounted rib, the detailed coherent structures present in the reattaching shear layer should be known. Hence, one goal of the present study is to explore the presence of coherent structures in the separated shear layer behind a wall-mounted rib and to identify the appropriate scaling parameters for these coherent structures. The efficacy of flow control techniques is dependent on the phase locking between the control signal and the flow. Therefore, the suitability of the control technique can be quantified by examining the phase jitter evolution in a flow. Ho et al (1991) used a technique referred to here as HZFB method for showing the phase decorrelation of coherent structures in free shear layers. An attempt is made here to evaluate the HZFB technique for the quantitative representation of the development of phase jitter, and based on this evaluation, a modified HZFB technique is suggested here to eliminate the effect of small scales in the phase jitter calculations. A pattern recognition technique is also proposed here for the phase jitter calculation. 
Wallace et al (1972) and Willmarth \& Lu (1972) used the quadrant analysis technique to identify the mechanisms associated with the production and transport of the shear stress in flat plate boundary layer and channel flows. In their analysis, the flow motion is represented by a sequence of events, i.e. ejection, sweep, outward interaction motion, and wallward interaction motion, and thus the turbulence production motion is partitioned into these four events. One of the goals of the present study is to explore the effect of excitation on the above sequence of turbulent motions.

\section{Description of experiment}

The schematic of the experimental set-up for the measurements is shown in figure 1 . Air is drawn into a rectangular channel of $0.3 \mathrm{~m}$ by $0.06 \mathrm{~m}$ cross section through a 5.25 to 1 contraction section, honeycomb and wire screens by a blower operating in suction mode. The channel is followed by a settling chamber designed to eliminate swirl and large-scale turbulence generated by the blower. For exciting the flow, an acoustic arrangement similar to that of Fiedler \& Mensing (1985) in their study of plane turbulent shear layer excitation is used. A loudspeaker is chosen for the flow excitation because this arrangement is simple and yields a repeatable range of amplitude and frequency. The two-channel PCI-312 signal generator from PC Instruments Inc. provides the required driving signal to the loudspeaker through a power amplifier from Velodyne Acoustics Inc. (Model OLD-12-N). The loudspeaker is connected to a rectangular cavity of dimension $0.5969 \mathrm{~m} \times 0.3048 \mathrm{~m} \times 0.0222 \mathrm{~m}$. The sound wave from this cavity enters the test section through a $0.00476 \mathrm{~m}$ wide slot that spans the width of the channel. Styrofoam is mounted on the wall of the cavity, opposite to the loudspeaker, to minimize the effects of multiple reflections of acoustic waves inside the cavity. Tam (1978), from the numerical study of acoustic excitation of instability waves in a two-dimensional shear layer, observed that a narrow beam aiming at an angle between $50^{\circ}$ and $80^{\circ}$ is most effective in exciting the instability waves. The aiming angle of the acoustic beam used here is $90^{\circ}$.

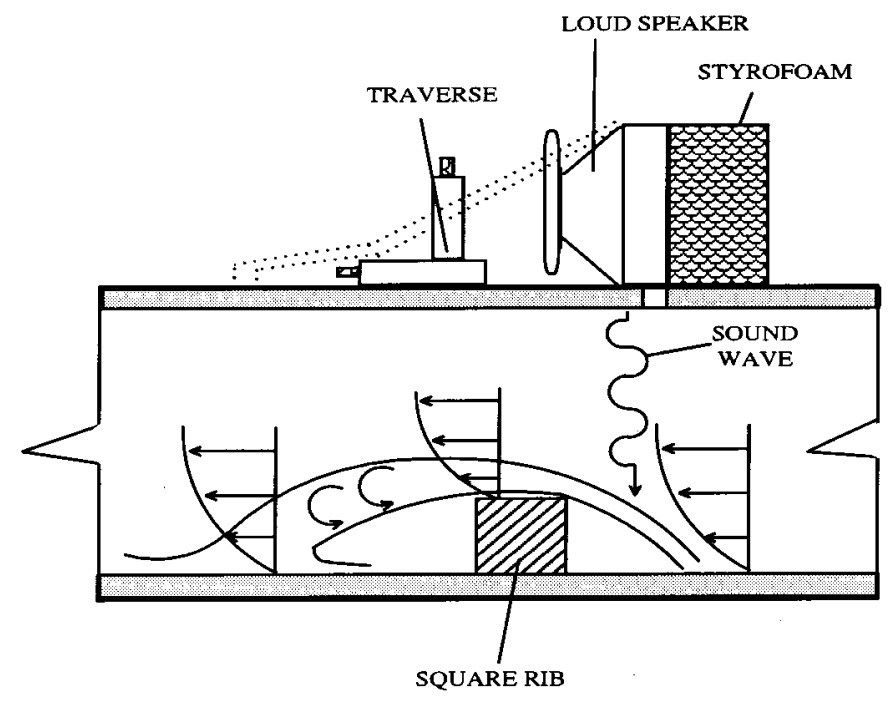

Figure 1. Schematic of the experimental set-up. 
A hot-wire anemometer was used for the velocity measurement. The anemometer was operated in the constant temperature mode with $70 \%$ overheat ratio. The anemometer output was connected to a Keithley Metrabyte data acquisition board (DAS-16) through a screw terminal accessory board (STA-16). The Streamer package supplied by Keithley Metrabyte was used for direct storage of data from the DAS-16 board to the hard disk of the computer. The hot-wire was calibrated using a Model 1125 calibrator supplied by TSI Inc. The hot wire measurements were made at or above the transverse location where $U / U_{\max }=0.65$, i.e. in the region where reversed flow intermittency is zero.

\subsection{Initial conditions}

The initial state of the boundary layer, i.e. boundary layer thickness $(\delta)$, displacement thickness $\left(\delta^{*}\right)$, momentum thickness $(\theta)$ and shape factor $\left(\delta^{*} / \theta\right)$ at different Reynolds numbers and at the rib position but with the rib removed from the channel, are shown in table 1. When the rib is submerged fully in the approaching boundary layer, there is an approaching velocity gradient at the top surface of the rib. When the rib is not submerged inside the boundary layer, the top surface of the rib sees a uniform approach velocity. From table 1, it is seen that the $6.35 \mathrm{~mm}$ rib is inside the boundary layer for $\operatorname{Re}=11400$ and 8200 , and outside the boundary layer for the $\operatorname{Re}=14600$ case. The next issue of interest is the state of the approaching flow. Shape factors $\left(\delta^{*} / \theta\right)$, presented in table 1 , are close to the shape factor of a $1 / 7$ th law turbulent layer i.e. $\simeq 1.22$ indicating that the flow is approaching turbulent flow. Velocity profiles are plotted at different Reynolds numbers with the corresponding laminar (Blasius) and turbulent values (law of the wall and $1 / 7$ th law profile). The almost perfect match with experimental values indicates that the approach flow is turbulent.

\subsection{Forcing criteria}

It is expected that both the forcing frequency and the forcing amplitude play an equally important role in the modification of large-scale structures. From the power spectral density evolution in the streamwise direction (see figure 2), it can be observed that the fundamental frequency is the dominant frequency observed in the near-field of the downstream edge of the rib, and represents the shear layer mode in the present flow and the subharmonic manifests in the downstream direction. These modes also have been reported to be the most dominant in simpler mixing layer studies. Thus, the excitation frequency selected for this investigation is equal to the fundamental frequency.

The forcing level is defined here as the ratio between the r.m.s. velocity of the largescale fluctuations attributed to the excitation and the average velocity measured at the

Table 1. Boundary layer characteristics at the bottom surface of the channel without the rib but at the same location as the rib.

\begin{tabular}{|c|c|c|c|c|}
\hline Reynolds No. & $\delta(\mathrm{mm})$ & $\delta^{*}(\mathrm{~mm})$ & $\theta(\mathrm{mm})$ & $\delta^{*} / \theta$ \\
\hline 14600 & 6.0 & 1.542 & 1.088 & 1.417 \\
\hline 11400 & 7.5 & 1.202 & 0.879 & 1.367 \\
\hline 8200 & 9.0 & 1.055 & 0.744 & 1.418 \\
\hline
\end{tabular}



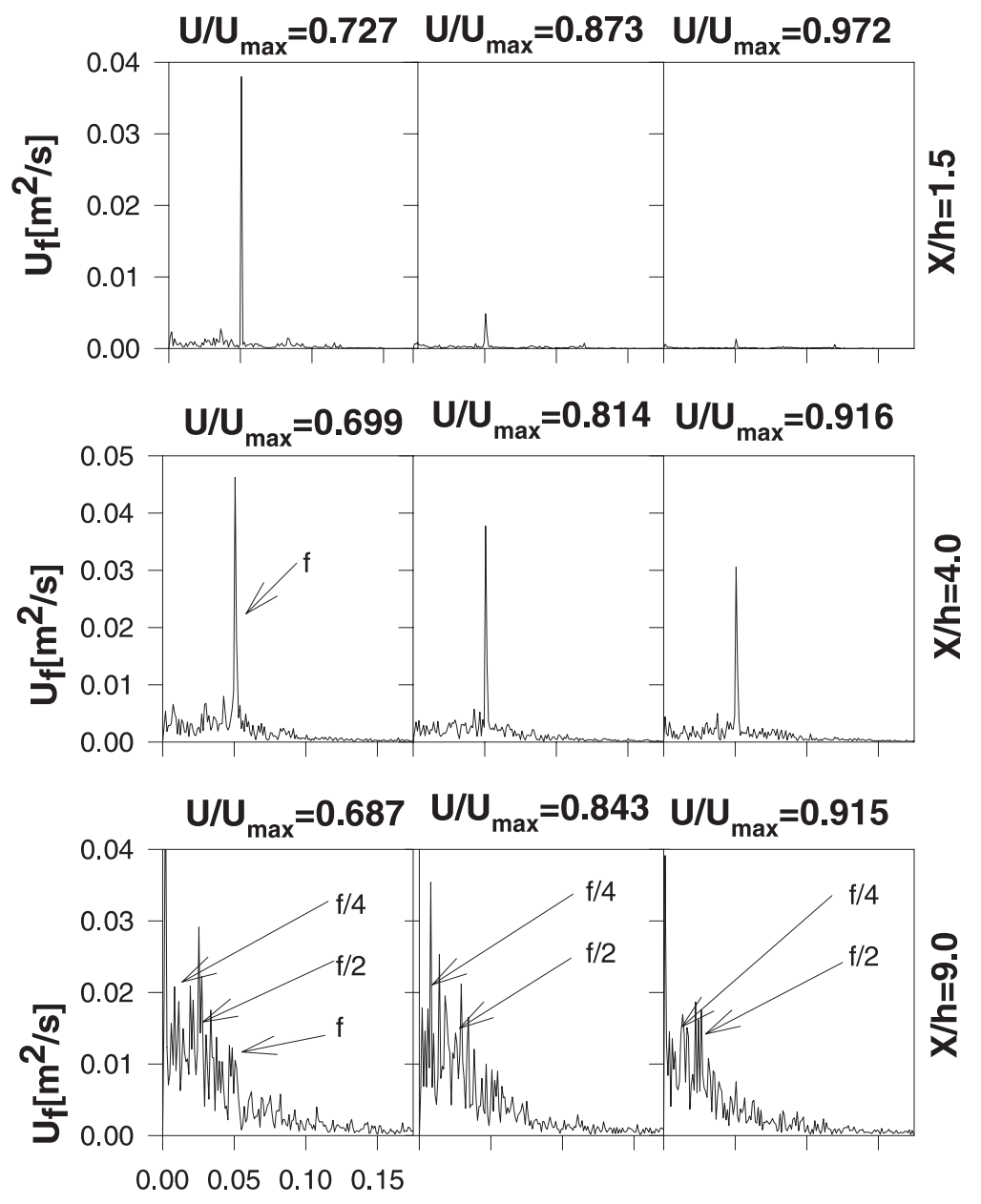

$\mathbf{f}(\mathbf{k H z})$

Figure 2. The spectral evolution in the downstream direction of the $6.35 \mathrm{~mm}$ rib at Reynolds number equal to 14600 .

downstream edge of the rib at a $Y / h$ distance equal to 1.0 from the rib surface. This location represents the external free stream at the downstream edge of the rib. Different forcing levels were obtained by adjusting the voltage input to the power amplifier of the loud speaker and thus the r.m.s. value of the $u$-velocity. For a desired amplitude and phase difference, the input to the function generator depends on the frequency response of the loud speaker chamber. Hence, initial calibration of the loud speaker was performed by plotting and curve fitting the forcing level versus the voltage supplied from the function generator. This helped in the selection of the correct input from the function generator to the power amplifier for a particular forcing frequency and forcing level. The momentum thickness at $X / h=8.5$ versus forcing level plot showed an increase in momentum thickness upto the forcing level of $2 \%$ and afterwards it remain almost constant indicating that $2 \%$ forcing level is the optimum one. Thus, the forcing level for the fundamental forcing case was selected here to be $2 \%$. 


\section{Data analysis procedure}

The instantaneous streamwise velocity component $(u(x, y, t))$ can be decomposed into the global mean field $(U(x, y))$, the periodic coherent field $\left(u_{c}(x, y, t)\right)$ and the random background turbulent field $\left(u_{r}(x, y, t)\right)$ (Hussain \& Zaman 1980), i.e.

$$
u(x, y, t)=U(x, y)+u_{c}(x, y, t)+u_{r}(x, y, t) .
$$

Using this kind of decomposition, equations for the velocity components have been devised for the mean, the periodic and the random turbulent velocity fields in the flow. The experimental measurements can be decomposed in the same fashion and the behaviour of the three components of the turbulent field can be analyzed. The following subsections discuss the techniques used here for determining the three components of the turbulent signal.

\subsection{Phase averaging method}

The most widely used triple decomposition technique for deducing the turbulent and organized-unsteady component of turbulent flow is the phase averaging method. The assumptions behind this technique is that the organized unsteady motion is identical from cycle to cycle and the random turbulent motions in successive cycles are independent motions (uncorrelated with one another). Here, the phase average or ensemble average is obtained from:

$$
\langle u(x, y, t)\rangle=\lim _{N \rightarrow \infty} \frac{1}{N} \sum_{n=0}^{N-1} u(x, y, t+n T),
$$

where $T$ is the period of the cycle. From the assumption that the phase averaged and turbulent components are uncorrelated in time, the following equations are used to calculate the large-scale and small-scale turbulent motions.

$$
\begin{aligned}
& u_{r}(x, y, t)=u(x, y, t)-\langle u(x, y, t)\rangle, \\
& u_{c}(x, y, t)=\langle u(x, y, t)\rangle-U(x, y) .
\end{aligned}
$$

The accuracy of the phase averaging method is dependent upon the accuracy with which the cycle period is known and the repeatability of the organized motion. If the time period is inaccurate, then the organized motion is reported as turbulence. Similarly, when the organized motion varies from cycle to cycle, the organized motion is reported as turbulence.

\subsection{Fourier method}

In the Fourier method, the large-scale vortices are assumed to be composed of several instability waves which propagate and amplify in the downstream direction. Thus the coherent velocity field is written as the superposition of dominant modes present in the velocity field i.e.,

$$
u_{c}(x, y, t)=\sum_{k} A_{k}(x, y) \cos \left(k \omega_{f} t+\phi_{k}(x, y)\right), k=1,2,3,4 \ldots
$$


where, $k$ refers to the predominant modes, $A_{k}(x, y)$ and $\phi_{k}(x, y)$ are the amplitude and phase angles of the respective modes. The amplitude and phase of the signal can be determined using Fourier Transform and the coherent contribution of different modes and the total coherent contribution can be determined. Compared to the phase averaging method, where only the identification of fundamental frequency is done, the Fourier method identifies the different predominant modes and thus provides a total picture of the large-scale structures. The advantage of the Fourier analysis is that it can be used to study different frequency structures. The limitation of the Fourier method is that it under-predicts the coherent magnitude and over-predicts the random magnitude in the presence of phase jitter in the signal.

\subsection{Pattern recognition technique}

In pattern recognition technique, the coherent velocity component is approximated to be the combined contribution from several modes. Based on the power spectral density distribution shown in figure 2, the possible dominant modes in such flows are the 5/2 fundamental, first harmonic, 3/2 fundamental, fundamental, first sub-harmonic, second sub-harmonic and third sub-harmonic. The coherent velocity component can then be written as:

$$
u_{c}(x, y, t)=\sum_{k} A_{k}(x, y) \cos \left(k \omega_{s} t+\phi_{k}(x, y, t)\right), k=1 / 4,1 / 2,1,2,3,4,5,
$$

where $\omega_{s}$ is the first sub-harmonic frequency (equal to half the forced frequency or natural frequency), $A_{k}$ and $\phi_{k}$ are the amplitude and phase corresponding to the respective mode of the wave in the velocity signal and $k=1 / 4,1 / 2 \ldots .5$ corresponds to the third sub-harmonic, second sub-harmonic, first sub-harmonic, fundamental, 3/2 fundamental, harmonic and 5/2 fundamental respectively. For modes having phase jitter, the $\phi_{k}$ values are not constant from one period of the mode to another. Any contribution from frequency larger than the maximum frequency (here the 5/2 fundamental) can be regarded as coming from the random small-scale motion. Any contribution below the minimum frequency considered (here the third sub-harmonic) appears in the zeroth coefficient of the Fourier series and represents the jitter of the mean velocity per segment.

The evaluation of the Fourier components for different modes is performed by segmenting the whole time series of the signal into different segments such that in one segment, one period of the corresponding wave is present. The total mean of the time series is first subtracted from the signal. Then the calculation of the Fourier components is performed starting with the lowest frequency, i.e. third sub-harmonic, using segments eight fundamental waves long. The amplitude and phase of the third sub-harmonic are calculated by using the traditional Fourier technique and then the contribution of the third sub-harmonic is subtracted from the time series for calculation of the next mode (i.e. second sub-harmonic). The amplitude and phase calculation of the second sub-harmonic uses segments four fundamental waves long and then the contribution of the second sub-harmonic is subtracted to obtain the new time series. This time series is then divided into segments of twice the fundamental period. This time period is then used to evaluate the $3 / 2$ fundamental, the $5 / 2$ fundamental and the subharmonic. For the $3 / 2$ fundamental and $5 / 2$ fundamental, segments 1.5 times the fundamental time period and 0.4 times the fundamental time period cannot be used, because the respective magnitude calculation will be affected by the fundamental wave. Segments equal to the 
fundamental time period also cannot be used as the segment will not contain whole numbers of waves of $3 / 2$ fundamental and 5/2 fundamental frequency. Therefore, a segment of length equal to twice the fundamental time period is used. The $3 / 2$ fundamental and $5 / 2$ fundamental wave amplitudes and phases are calculated first from these segments and then their contributions are subtracted from the time series before computing the first subharmonic. After subtracting the contribution of the first sub-harmonic, the calculation of the fundamental and first harmonic is performed using segments of length equal to one period of the fundamental wave. Finally, the coherent structure amplitude is calculated by summing the amplitudes of all modes (5/2 fundamental, first harmonic, 3/2 fundamental, fundamental, first sub-harmonic, second sub-harmonic and third sub-harmonic).

\subsection{Phase jitter using the HZFB technique}

In the HZFB technique (Ho et al 1991), the period of the coherent structure passage is calculated from the fluctuating velocity signal as the distance between the positively sloped zero crossing points. These time periods of structure passage are then used to construct the histogram of normalized time. The log-normal probability distribution is used to curve fit the histogram. The standard deviation of the log normal distribution provides the measure of the magnitude of the phase jitter in the signal. The log-normal distribution used is given by:

$$
P(\tau)=\frac{1}{(2 \pi)^{0.5} \sigma \tau} \exp \left[-\frac{1}{2}\left(\frac{\ln (\tau)-\mu}{\sigma}\right)^{2}\right],
$$

where $\sigma$ is the standard deviation and $\mu$ is the mean value of $\ln (\tau)$ and $\tau$ is the non-dimensional period calculated by normalizing the period of each coherent structure $(T)$ by the local average time period. In the presence of two peaks in the histogram, the sum of two log-normal distributions are used with four parameters such that the area under the fitted histogram is equal to 1 . The final equation of the combined log-normal distribution, describing the two peaked probability density distribution is:

$$
\begin{aligned}
P(\tau)= & \frac{1}{(2 \pi)^{0.5}\left(\sigma_{1}+C \sigma_{2}\right) \tau}\left[\exp \left[-\frac{1}{2}\left(\frac{\ln \tau-\mu_{1}}{\sigma_{1}}\right)^{2}\right]\right. \\
& \left.+C \exp \left[-\frac{1}{2}\left(\frac{\ln \tau-\mu_{2}}{\sigma_{2}}\right)^{2}\right]\right],
\end{aligned}
$$

where, $\mu_{1}$ and $\mu_{2}$ are the $\tau$ value at first and second peak, while $\sigma_{1}$ and $\sigma_{2}$ are the standard deviations of the respective peaks and $C$ is an arbitrary constant. The total standard deviation of the above histogram is calculated by using the following expression:

$$
\sigma^{2}=\frac{\sigma_{1}}{\sigma_{1}+C \sigma_{2}}\left(\sigma_{1}^{2}+\mu_{1}^{2}\right)+\frac{C \sigma_{2}}{\sigma_{1}+C \sigma_{2}}\left(\sigma_{2}^{2}+\mu_{2}^{2}\right)-\left(\frac{\sigma_{1}+\mu_{1}+C \sigma_{2} \mu_{2}}{\sigma_{1}+C \sigma_{2}}\right)^{2},
$$

where, $\sigma$ is the total standard deviation of the two peaked probability distribution.

It is observed from the simulated data and the reattaching shear layer data that the HZFB method gets affected due to the presence of multiple modes in large-scale structures and random small scales. Considering the above limitations, in the following sections two new techniques for phase jitter calculation are suggested. One of the methods is the 
modification of the HZFB method to eliminate the effect of small scales in the phase jitter calculation. The other method is based on the pattern recognition technique, which provides the phase jitter magnitude in the individual modes of the forcing signal.

\subsection{Phase jitter using modified HZFB method}

In the HZFB method, the positively sloped zero crossing points are calculated on the assumption that if the fluctuating velocity signal changes sign from the $(k-1)$ to the $k$ th point and the $(k+1)$ th point signal is larger in magnitude than the $k$ th point, then the $k$ th point is assumed to be a valid zero crossing point with positive slope. Using this criteria the method is successful in predicting the phase jitter of simulated data consisting of random noise. However, for the reattaching shear layer, the results obtained using the above criteria showed high probability density for small time periods (close to $\tau \simeq 0$ ), indicating that the histogram includes the periods of the small scale structures. This is undesirable since the primary idea behind the HZFB method is to delineate the phase decorrelation of large scale structures. Hence, an attempt is made here to modify the HZFB method to exclude the small-scale periods in the population of large-scale periods. The method used is discussed in the following paragraph.

The basic idea behind this method is that a valid zero crossing point is decided not only on the basis of the slope at zero crossing but also on the basis of minimum number of positive points after the zero crossing. If the required minimum number of positive points (first parameter) are specified to be small then small-scale time periods are included; if it is large, only the more energetic eddies corresponding to the large-scale time periods are included. This parameter therefore controls the range of scales to be included in the coherent structure classification. This parameter is decided based on the highest frequency that can be considered as a frequency of coherent structures or large scales. It can be specified as the percentage of the total number of data points in a fundamental cycle (natural frequency or forcing frequency), that have to be positive in order to be considered a valid zero crossing point. The total number of points in a fundamental cycle is determined from the sampling rate and fundamental frequency. In performing the calculations, the required minimum number of positive points is incremented by one and the total number of zero crossing points are recalculated. The per cent difference between the previous number of zero crossing points and the recent number of zero crossing points is calculated. If it (the per cent difference) is less than that from some previously set value (this is a second parameter that has to be specified), it is assumed that the convergence of the procedure is obtained. The present population of time periods then consists of only large scales and the small scale effects are negligible.

\subsection{Phase jitter using pattern recognition technique}

In this approach, the phase jitter present in the large-scale structure is assumed to be predominantly from the discrete large-scale modes considered. For a single-frequency forcing, the jitter manifests in the forcing wave frequency itself. For the two-mode forcing, (i.e. fundamental and first-sub-harmonic) the phase jitter may be present in either of the modes or in both of them. Hence, in the pattern recognition method, phase jitter is calculated for both the fundamental and the first sub-harmonic and in the phase difference between the two modes. The phase difference $(\Delta \phi)$ between the two is calculated as 


$$
\Delta \phi=2 \phi_{s}-\phi_{f},
$$

where $\phi_{s}$ is the phase angle of the first sub-harmonic and $\phi_{f}$ is the phase angle of the fundamental. A histogram is constructed from the whole population of phase differences $\left(\phi_{k}\right)$ computed using the above described pattern recognition procedure for coherent amplitude calculation. The mean and standard deviation of the histogram is calculated by fitting the histogram with a normal distribution using the least squares method. The standard deviation of this distribution is used as a quantitative measure of the phase jitter in the turbulent signal.

\section{Results and discussions}

Results obtained in this work are discussed in the following sequence. The large-scale structures present behind the surface-mounted rib are explored using the spectral density and autocorrelation. The proper length scale for the calculation of the Strouhal number is investigated next. Subsequently, the data analysis techniques discussed and proposed in this paper are tested using the simulated data and the reattaching shear layer data. Magnitude of the large-scale structures and the associated phase jitter of the flow behind the surface-mounted rib are presented and discussed next. Finally, the types of turbulent motions i.e. ejection, sweep, outward interaction motions and wallward interaction motions, are presented with the help of the quadrant analysis technique.

\subsection{Spectral density and autocorrelation}

In this section, autocorrelation and power spectral density are presented at selected locations to verify the presence of large-scale vortices in the reattaching shear layer. Autocorrelation graphs of velocity data for Reynolds number 14600 is shown in figure 3 . The presence of a sinusoidal variation in the autocorrelation function at $Y / h=1.404$ is clearly evident. Since autocorrelation of a sinusoidal function is sinusoidal (Hinze 1975), the sinusoidal variation of the autocorrelation function indicates that there is a definite vortex shedding present in the flow. Along the lower side of the shear layer $(Y / h=0.78$ and 1.092), the autocorrelation plots are representative of random turbulence. When the upper side of the shear layer $(Y / h=1.716)$ is approached, higher harmonics of the vortex shedding frequency show up in the autocorrelation function.

The downstream spectral evolution of the flow past the $6.35 \mathrm{~mm}$ rib at Reynolds number equal to 14600 is shown in figure 2. The reattachment length for this case is about $5.5 h$. Therefore, the $X$-location shown includes the near field region $(X / h=1.5)$, the near reattachment region $(X / h=4.0)$, and the past reattachment region $(X / h=9.0)$. It may be noted that upstream of reattachment, a single-mode instability (the fundamental, $f$ ) is clearly observed but after reattachment, the magnitude of the subharmonic $(f / 2)$ is predominant. The energetic eddy (at the fundamental $f$ ) is observed to grow in the downstream direction (increasing $X / h$ ) in the near field region, and the growth rate is not same at different $Y / h$ location. Differences in the amplitude of the spectrum in the wall normal $(Y)$ direction indicates that effect of the coherent wave is clearly present in core of the shear layer and weakens at other wall normal locations. This behaviour is consistent with the nature of convective instabilities present in shear layers (Huere $\&$ Monkewitz 1985). The emergence of the subharmonic at $X / h=9$, points to the possibility of vortex pairing or merging downstream of reattachment. 


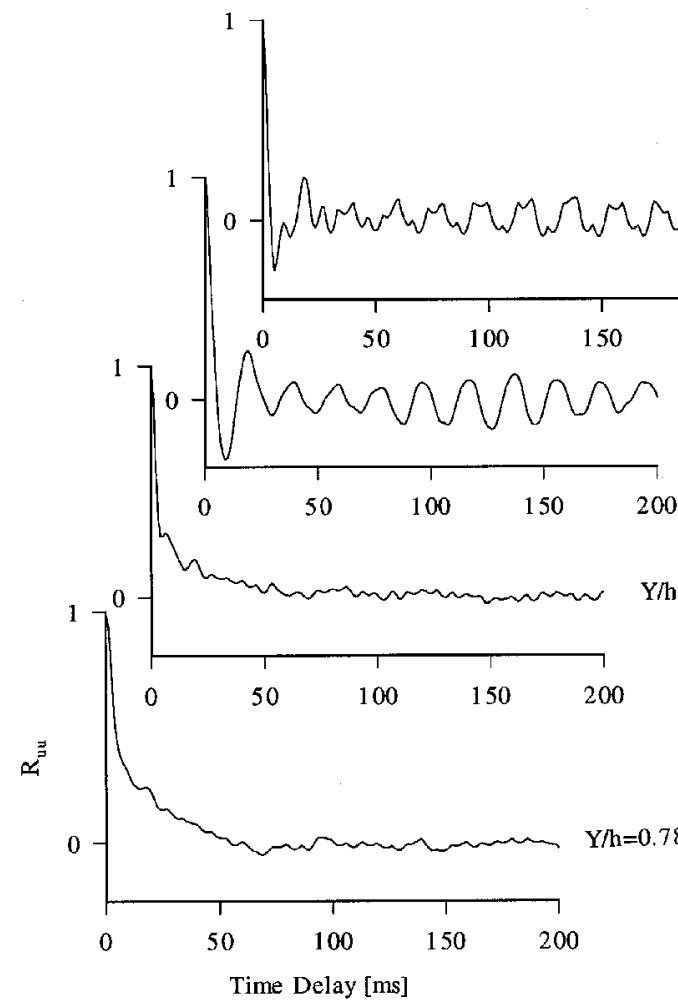

Figure 3. Autocorrelation behind $6.35 \mathrm{~mm}$ rib at $X / h=2$ and at Reynolds number $=14600$.

\subsection{Length scale for Strouhal number calculation}

The values of the most unstable frequency for different sized ribs and the Strouhal number based on the rib height were calculated and it was observed that the Strouhal number (based on rib height) is not constant as opposed to that reported for ribs placed in the free stream. It varies within the range of 0.079 to 0.381 which is significantly different from the value of 0.12 to 0.2 reported for the rib in a free stream case. These results indicate that the rib height is not the true length scale for scaling the vortex shedding frequency of the surface-mounted rib. Arnal et al (1991) used a length scale of $0.5 \mathrm{~h}$ for a rib in the free stream and $h$ for a rib fixed on the wall and observed that the Strouhal number lies around 0.072. They concluded that length scale of vortex dynamics is different for the free stream and wall-bounded cases. For a wall-bounded case, they argued that vortices scale with $h$ whereas for a free stream case, they scale with $h / 2$. Their conclusions are not however supported by this experimental investigation. According to this investigation, the Strouhal number for a surface-mounted rib, do not scale with either $h$ or $h / 2$. This discrepancy may be attributed to the fact that the calculations reported by Arnal et al (1991) are for laminar flow, while the actual flow is turbulent. In the following paragraphs, the true length scale for the Strouhal number calculation of the surface-mounted rib is explored.

The Strouhal numbers are calculated next based on the momentum thickness $(\theta)$ and vorticity thickness $\left(\delta_{w}\right)$ at the downstream edge of the rib. The velocity at the downstream edge of the rib away from its surface increases to a maximum value and then decreases. The boundary layer thickness $(\delta)$ at the downstream edge of the rib for the calculation 
of momentum thickness and vorticity thickness, is defined as the distance from the rib surface where the velocity in the shear layer reaches a maximum value. Negative velocities are observed at some $Y / h$ locations at the downstream edge of the rib. It is observed that the momentum thickness calculated using both positive and negative velocities at the point of separation is not the true length scale for vortex shedding as the Strouhal number has wide variation. Therefore, the momentum thickness are calculated next excluding the negative velocity at the downstream edge of the rib which is shown in table 2.

The Strouhal number based on the momentum thickness is calculated by using both $\bar{U}$ and $U_{\mathrm{Av}}$ as characteristic velocity where, $\bar{U}$ is the difference between the maximum and minimum velocity and $U_{\mathrm{Av}}$ is the average velocity at the downstream edge of the rib. It is observed that the Strouhal number based on momentum thickness (calculated excluding the negative velocities at downstream corner of the rib) and $U_{\mathrm{Av}}$ is fairly constant (in the range of 0.01-0.019) with an average value of 0.013. Strouhal numbers based on the momentum thickness and $\bar{U}$ are within the range 0.014 to 0.04 . Mcmanus et al (1990) studied shear layer excitation of both reacting and non-reacting flow of a backward-facing step. Strouhal numbers based on momentum thickness for non-reacting flow $(\mathrm{St}=f \theta / \bar{U})$ were 0.026 and 0.034 and within the range suggested by Ho \& Huerre (1984) $(\mathrm{St}=0.032)$ for the natural hydrodynamic instability. Hasan (1992) observed that for a backward-facing step, non-dimensional natural instability frequency of the reattaching shear layer scales with momentum thickness $(\theta)$ rather than with the step height and is about 0.012. Thus, the non-dimensional number based on momentum thickness calculated excluding the negative velocity is consistent with the results of the backward-facing step by Hasan (1992) and Mcmanus et al (1990) and mixing layer by Ho et al (1984). Vorticity thickness is found to be same whether negative velocities are excluded or included in its calculations. This indicates that the maximum velocity gradient

Table 2. Strouhal number based on momentum thickness and vorticity thickness at point of separation $(X / h=0)$. Negative velocities at point of separation were excluded in the calculation of momentum thickness.

\begin{tabular}{rrrrccc}
\hline$h[\mathrm{~mm}]$ & $\operatorname{Re}\left(D_{h}\right)$ & $\theta[\mathrm{mm}]$ & $\delta_{\omega}[\mathrm{mm}]$ & $f_{m} \theta / U_{\mathrm{Av}}$ & $f_{m} \theta / \bar{U}$ & $\pi f_{m} \delta_{\omega} / 2 \bar{U}$ \\
\hline 3.17 & 14600 & 0.627 & 1.232 & 0.016 & 0.034 & 0.109 \\
3.17 & 11400 & 0.701 & 1.563 & 0.018 & 0.038 & 0.091 \\
3.17 & 8200 & 0.781 & 1.44 & 0.019 & 0.041 & 0.124 \\
6.35 & 14600 & 0.472 & 1.395 & 0.012 & 0.020 & 0.098 \\
6.35 & 11400 & 0.550 & 1.389 & 0.010 & 0.017 & 0.068 \\
6.35 & 8200 & 0.691 & 1.968 & 0.015 & 0.025 & 0.121 \\
9.52 & 14600 & 0.366 & 1.301 & 0.014 & 0.014 & 0.079 \\
9.52 & 13000 & 0.442 & 1.352 & 0.010 & 0.016 & 0.080 \\
9.52 & 11400 & 0.425 & 1.305 & 0.010 & 0.016 & 0.080 \\
9.52 & 9800 & 0.438 & 1.497 & 0.010 & 0.017 & 0.092 \\
9.52 & 8200 & 0.695 & 1.361 & 0.008 & 0.023 & 0.075 \\
15.87 & 14600 & 0.548 & 3.128 & 0.014 & 0.023 & 0.210 \\
15.87 & 11400 & 0.581 & 2.388 & 0.010 & 0.015 & 0.103 \\
15.87 & 8200 & 0.601 & 2.013 & 0.012 & 0.018 & 0.097 \\
\hline
\end{tabular}


is present outside the negative velocity region and the vortex shedding is influenced by the shear layer outside the negative velocity region. Therefore, it can be argued that the momentum thickness calculated including only positive velocities $\left(\theta^{+}\right)$at the downstream corner of the rib, is the most appropriate length scale for interpreting the non-dimensional frequency of vortex shedding behind the rib.

To assess the consistency of the current vortex shedding results, with the stability analysis, the values of $\omega^{*}$ at $X / h=0.0$ is calculated and summarized in table 2 , where, $\omega^{*}$ is the non-dimensional frequency based on vorticity thickness defined as:

$$
\omega^{*}=\pi f_{m} \delta_{w} / 2 \bar{U}
$$

From table 2 at $X / h=0.0$ (separation point) it is observed that $\omega^{*}$ is about 0.09 . Najjar et al (1993) calculated the non-dimensional frequency $\left(\omega^{*}\right)$ of a normal flat plate at a streamwise distance equal to 0.0 of the reattachment length and found it to be equal to 0.06 . Thus, the non-dimensional frequency based on vorticity thickness results of a surface-mounted rib is same order of magnitude as the observation of Najjar et al (1993) for a normal flat plate.

\subsection{Coherent structure amplitude}

To determine the effectiveness of the pattern recognition technique and the traditional Fourier method in the calculation of the coherent structure amplitude, simulated data consisting of different modes and phase jitter are first used. In an actual experiment, data representing large-scale structures with phase jitter can be modelled as a fundamental wave with phases normally distributed around the mean phase angle of the wave. The simulated signal, consisting of a fundamental wave with normally distributed phase, and the first, second and third sub-harmonics, is presented in figure 4 along with the reconstructed plots obtained from the pattern recognition and the Fourier method. The reconstructed signal from the pattern recognition method is almost on the top of the original signal, while the reconstructed signal from the Fourier method is considerably off with a maximum deviation of $63.18 \%$ in the coherent structure amplitude.

The performance of the pattern recognition technique is evaluated next using the velocity data of the reattaching shear layer developing behind the surface-mounted rib. The postreattachment region $(X / h=11.0)$ is selected and figure 5 shows the measured signal, and reconstructed coherent and random components of the signals calculated using the pattern recognition and Fourier methods. The pattern recognition method is successful in representing the coherent structure part indicating that the pattern recognition method is not affected in presence of phase jitter contrary to the Fourier method. The Fourier method under-predicts the coherent amplitude to a large extent and indicates that most of the turbulence contribution is due to random or small scales. However, from the results obtained using the pattern recognition method, it is observed that there is strong presence of large-scale structure well past the reattachment point $(X / h=11)$. It may be noted that similar observations about large-scale structures being present well past reattachment were made by Panigrahi \& Acharya (1999) using the octant analysis technique. In the reattaching shear layer, where there is appreciable phase jitter in the downstream direction, the pattern recognition technique is able to predict the coherent scale amplitude correctly as compared to the Fourier method. 


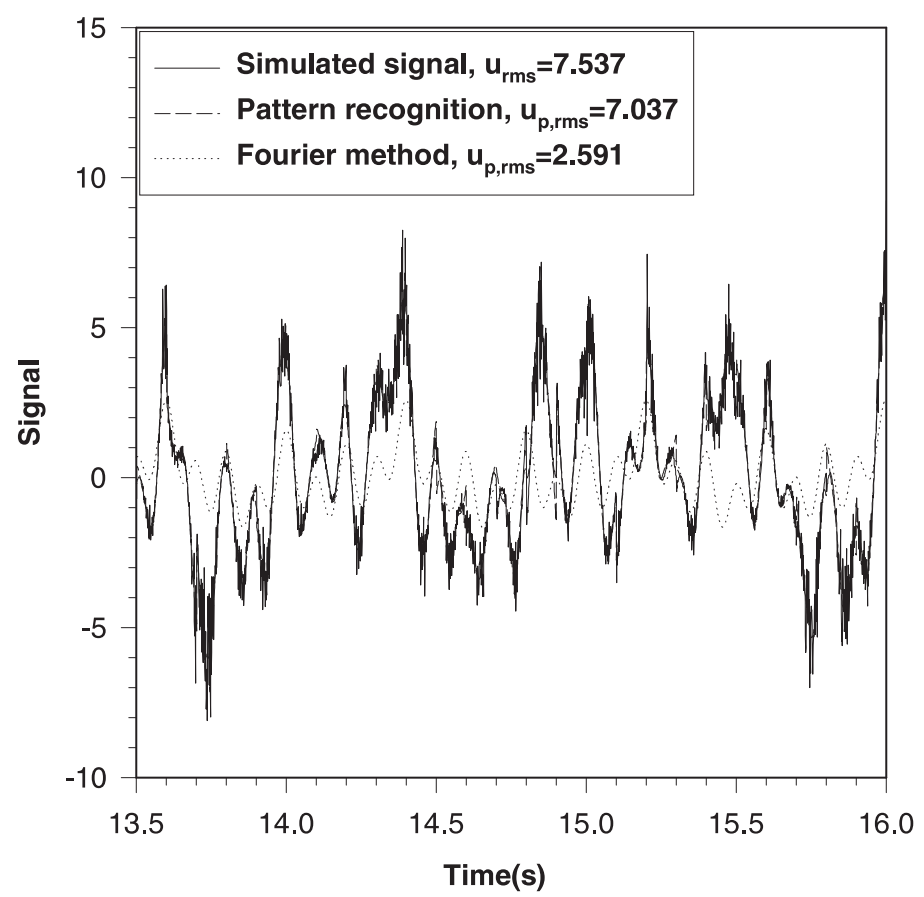

Figure 4. Simulated signal having fundamental, first sub-harmonic, second sub-harmonic and third sub-harmonic with phase variation normally distributed between $+\pi$ and $-\pi$ for the fundamental frequency, and the superimposed random scale with the reconstructed signal obtained using pattern recognition and Fourier method.

\subsection{Coherent structure phase jitter}

The performance of the HZFB method and the pattern recognition method are evaluated first using simulated data. All simulated data files used here have a random noise superimposed on top of the large-scale fluctuation with its amplitude varying randomly within $\pm 25 \%$ of the large-scale amplitude. The phase jitter is introduced into the simulated signal by assuming that the absolute phase $\left|\left(\phi_{k}(t)\right)\right|$ of a particular mode has a normal distribution about its mean, i.e. for each segment, the phase differences from segment to segment are random with a normal probability distribution. In figure 6 , the probability density distribution, obtained using the HZFB technique, of a simulated signal consisting of a fundamental wave with constant phase (phase jitter $=0$ ) throughout the entire time duration can be seen. The nature of the probability density distribution is similar to that in the near flow region of a forced mixing layer (Ho et al 1991). The above simulated data was also analysed with the pattern recognition method (see figure 7). In both figures 6 and 7, there is a steep peak with negligible standard deviation indicating the absence of phase jitter in the signal. Hence, both the HZFB and the pattern recognition methods predict the phase jitter value well for a signal having a constant phase fundamental wave with random noise superimposed on it.

To verify the ability of these techniques to predict the evolution of phase jitter, two simulated data sets consisting of a fundamental wave with the random phase normally distributed between $+45^{\circ}$ and $-45^{\circ}$ and between $+90^{\circ}$ and $-90^{\circ}$ are used. It is expected 


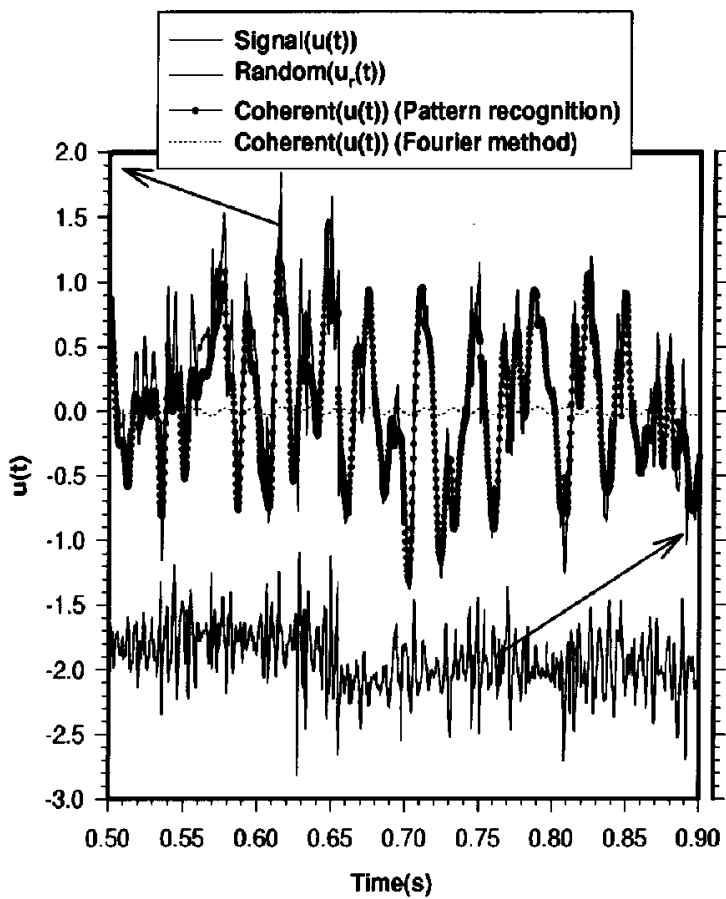

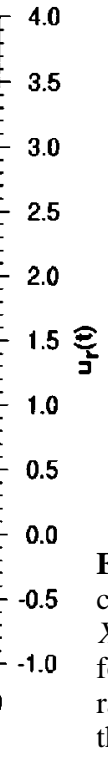

Figure 5. The coherent and random component of the velocity signal at $X / h=11.0$ at $U / U_{\max }=0.7$ location for forced reattaching shear layer. The random part $\left(u_{r}(t)\right)$ is obtained using the pattern recognition method.

that travelling waves with $\pm 45^{\circ}$ phase jitter will evolve into $\pm 90^{\circ}$ phase jitter signals and the ability of the two methods to track this phase decorrelation development should be evaluated. The results obtained by using the HZFB technique is shown in figure 6 . It is observed that for the $\pm 90^{\circ}$ phase jitter case, the standard deviation or phase jitter

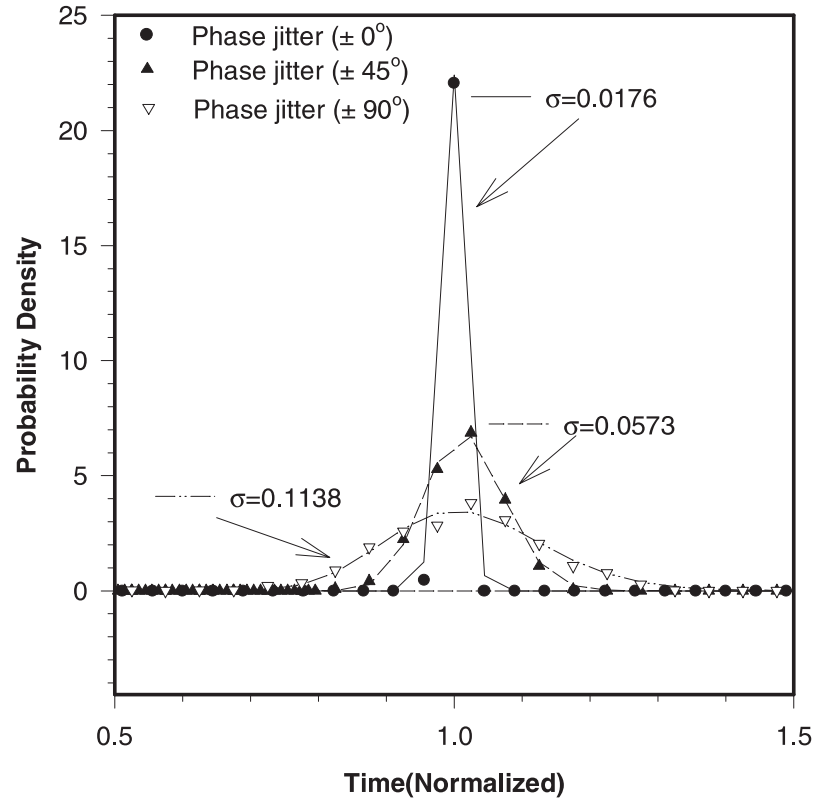

Figure 6. Results from the HZFB method using a simulated signal consisting of fundamental waves with and without phase jitter and random noise superimposed on it. 


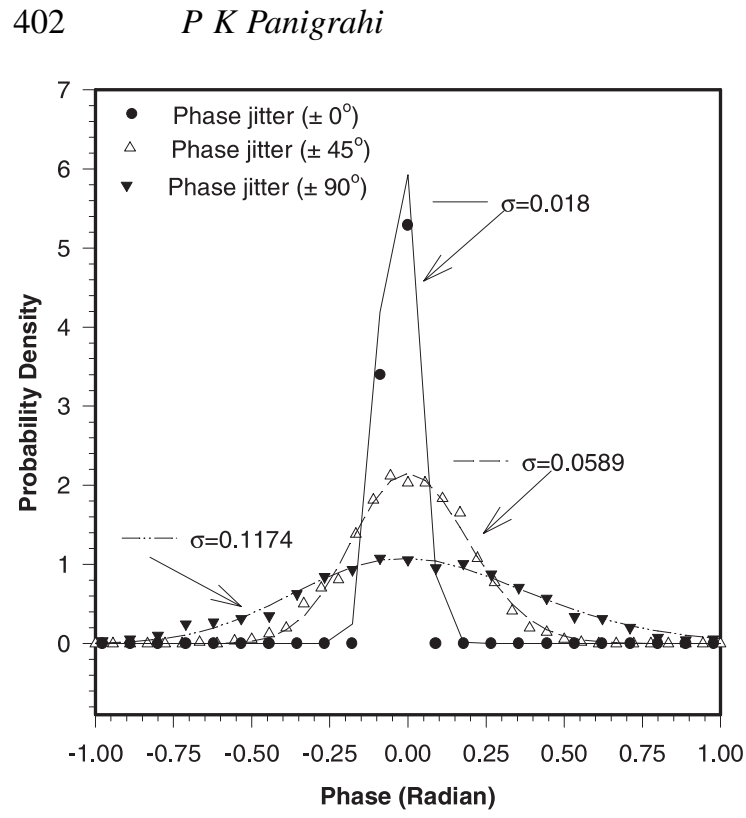

Figure 7. Results from the pattern recognition technique using a simulated signal consisting of fundamental waves with and without phase jitter and random noise superimposed on it.

increases in comparison to $\pm 45^{\circ}$ phase jitter case by $98.6 \%$ i.e. from 0.0573 to 0.1138 (see figure 6). When using the pattern recognition technique, it is observed that the standard deviation increases by $99.3 \%$ i.e. from 0.0589 for the $\pm 45^{\circ}$ case to 0.1174 for the $\pm 90^{\circ}$ case (see figure 7). Hence, it may be concluded that both the HZFB method and the pattern recognition technique work well in predicting the evolution of phase jitter for a turbulent signal with a single fundamental mode having random noise and random phase jitter from cycle to cycle.

Next, the reattaching shear layer data at the $U / U_{\max } \simeq 0.7$ locations is used to predict the phase jitter evolution during the shear layer development. The results from the HZFB method and the pattern recognition method are presented in figures 8 and 9 respectively. From the HZFB method (figure 8 ) it may be observed that the complete phase decorrelation takes place before $X / h=2.0$, long before the reattachment point $(X / h \simeq 4.0)$. In figure 10 , a single-mode frequency is observed to be predominant at $X / h=1.5$. The spectrum has a sharp peak without any side lobes indicating a very small presence of phase jitter. Therefore, the results from the HZFB method shown in figure 8 may be erroneous. The pattern recognition method (figure 9) shows complete phase decorrelation to occur after $X / h=3.0$. Power spectral density shown in figure 10 shows wider spectral distribution at $X / h=4.0$ and $U / U_{\max }=0.7$, supporting the phase jitter results obtained using pattern recognition method (figure 9), i.e. the presence of significant amount of phase jitter in the signal after $X / h=3.0$. The differences in the prediction between HZFB and pattern recognition technique cannot be linked to the presence of multiple modes, because from the power spectrum (figure 10), the multiple modes are not seen to be predominant before $X / h=3.0$. This implies that the presence of small scales may be responsible in affecting the results of the HZFB method. In figure 8, small scales, i.e. probability density at $\tau \simeq 0$ can be seen at $X / h>1.0$, supporting the above claim that small scales are dominant. Keeping this in mind, a modified HZFB method (described in data analysis section before) was suggested to eliminate the effect of small scales in the phase jitter calculation. 


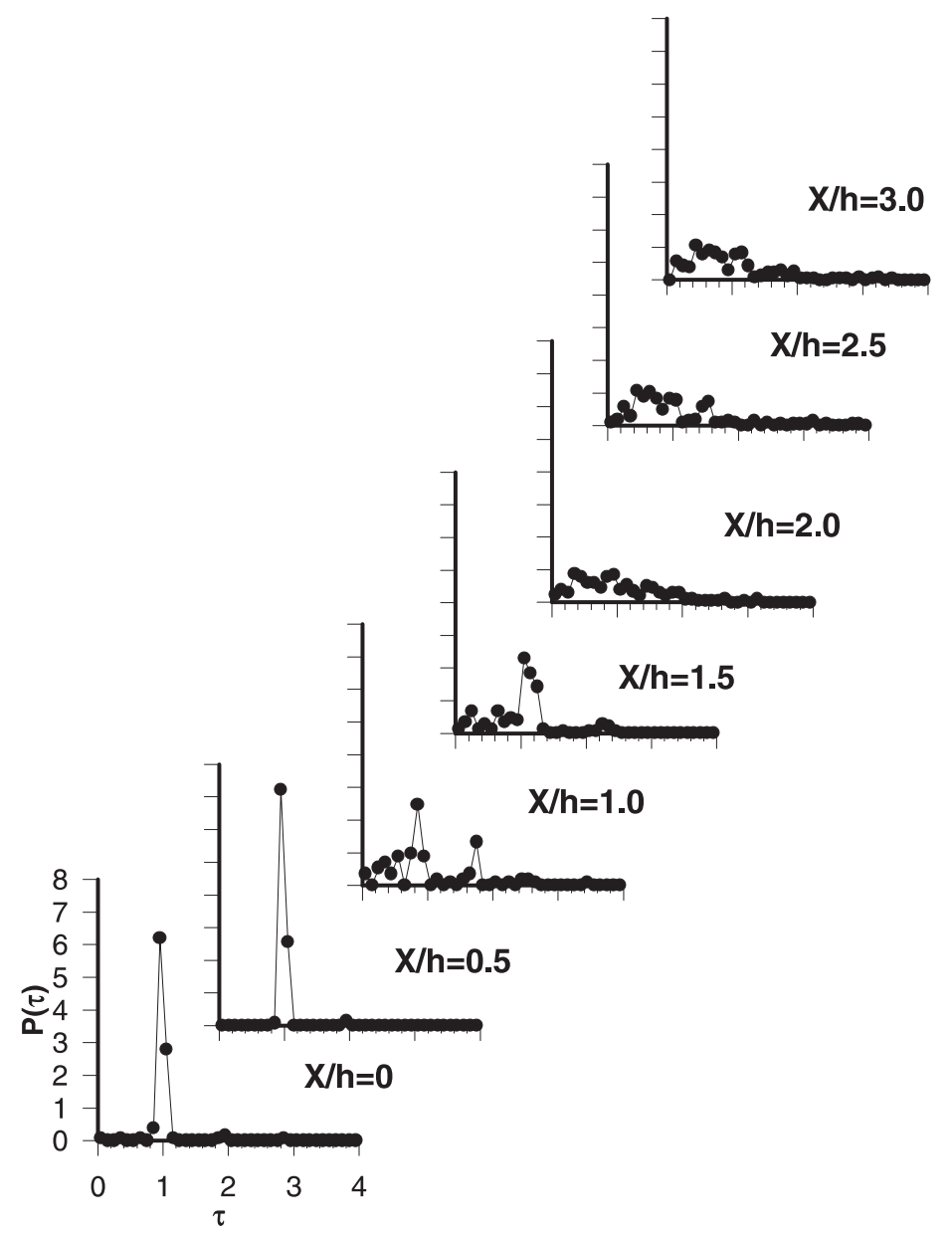

Figure 8. The phase jitter development of a forced reattaching shear layer forced at its natural frequency $=52 \mathrm{~Hz}$, along $U / U_{\max } \simeq 0.7$ using $\mathrm{HZFB}$ method.

The results obtained using the modified HZFB method are presented in figure 11 . Comparing figure 11 with figure 9, it can be observed that both the modified HZFB and the pattern recognition approaches give similar results. Both methods predict that complete phase decorrelation takes place at about $X / h=3.0$. Both the methods also predict slight reduction in phase jitter after the separation point $(X / h=0.0)$ implying improved organization of the structures and subsequent gradual increase in phase jitter till complete phase jitter takes plate at about $X / h=3.0$. The drawback of the modified HZFB method is that an a priori decision on the relative amplitudes and frequencies of the large scales has to be made. In the modified HZFB analysis, the parameters are chosen here such that the probability density near $\tau=0$ is close to zero, as they represent the time scales of random part. We tried to verify if the modifications made in the HZFB method to eliminate the effect of small-scale on phase jitter calculation has any effect on the large-scale structures. Therefore, the results presented previously by using HZFB method with simulated signal (figure 6) was reprocessed by using the modified HZFB method and no difference in results between the two was observed. This indicates that the results of the reattaching shear layer when using HZFB method are possibly 


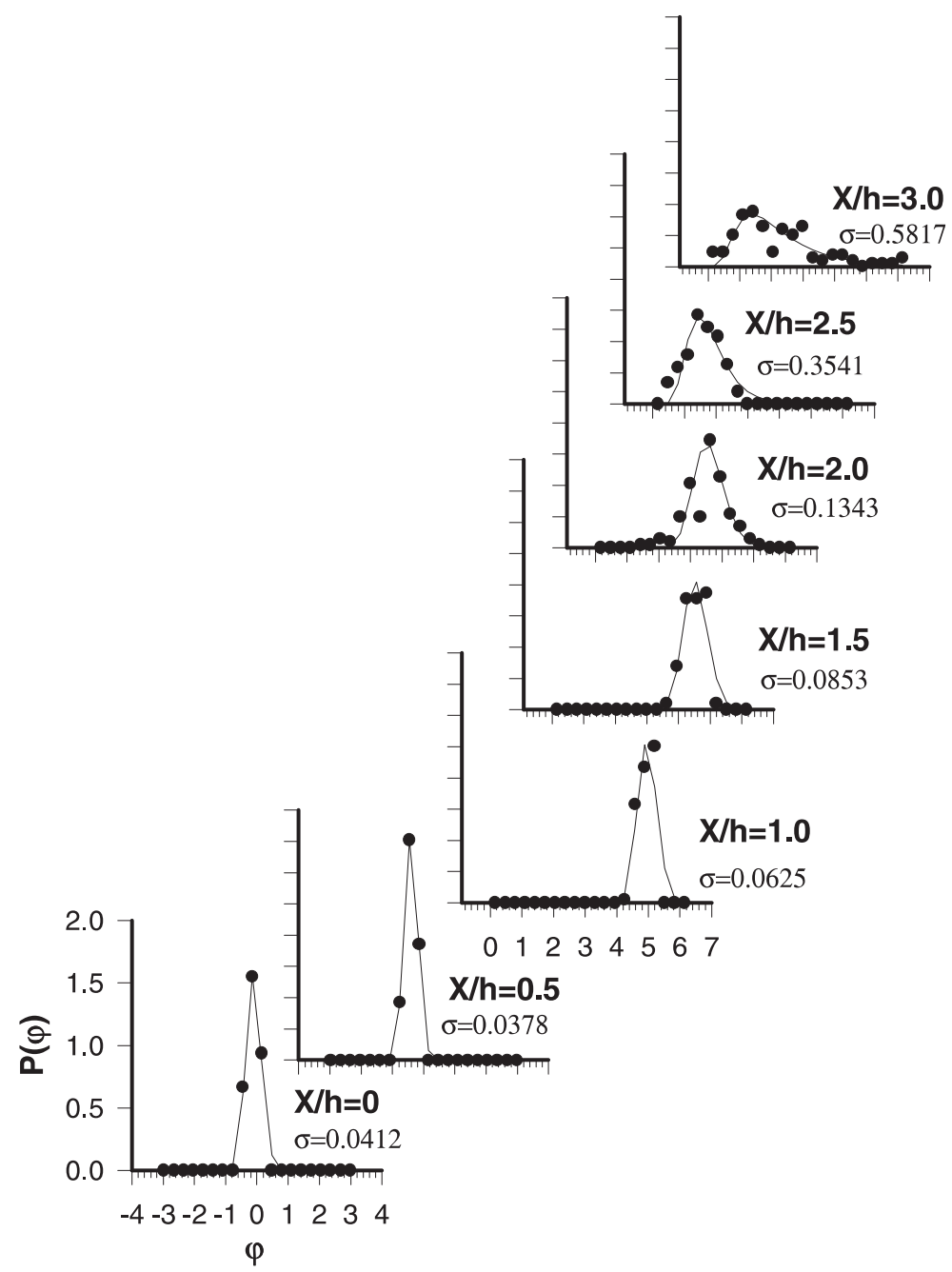

Figure 9. The phase jitter development of a forced reattaching shear layer forced at its natural frequency $=52 \mathrm{~Hz}$, along $U / U_{\max } \simeq 0.7$ using pattern recognition method.

affected by the small-scale turbulent structures only and the modified HZFB method is successful in predicting the reattaching shear layer phase jitter evolution. Therefore, it may be concluded that both the pattern recognition technique and the modified HZFB method are successful in correctly predicting the phase jitter in a forced reattaching shear layer.

The phase jitter evolution for the unforced case obtained using the modified HZFB method is shown in figure 12. Comparing with the results for the excited case shown in figure 11 it can be observed that excitation organizes the flow, because the phase jitter value is very small in the near field region of the excited flow in comparison to that for the natural flow. The phase jitter development in a reattaching shear layer, for both excited and unexcited case obtained using modified HZFB method is compared in figure 13. In the near field region, the phase jitter magnitude drops to a large extent when external excitation is applied. However, the steep increase in phase jitter value takes 

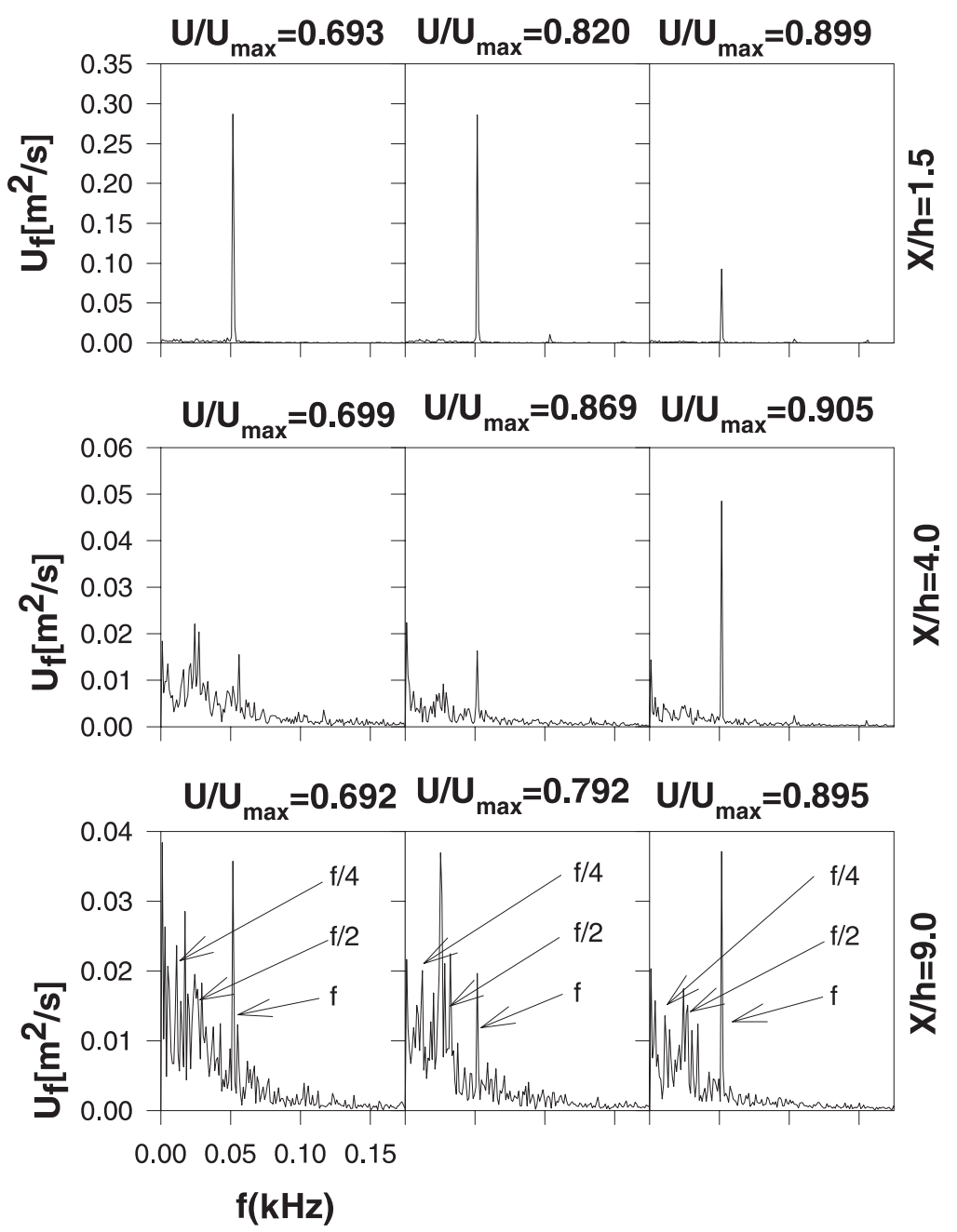

Figure 10. The evolution of the power spectral density of the $u$-velocity component for excitation at the fundamental frequency of $52 \mathrm{~Hz}$.

place at an earlier location for the excited flow in comparison to that of the unexcited flow. The reattachment length for the unexcited flow is about $5.5 h$, whereas for the excited flow it is about $4.0 \mathrm{~h}$. Thus, it can be inferred that the location of complete phase decorrelation of a reattaching shear layer is related to the reattachment region. The complex sweep and interaction motions at the reattachment point influences the organization of the coherent structure modes present in the shear layer resulting in increase of phase jitter value.

\subsection{Quadrant analysis}

The quadrant analysis technique is used here to investigate the types of turbulent motion present and to see the effect of excitation on the total contribution and scales of different turbulent motions both in the downstream and the cross-stream directions. The measurements 


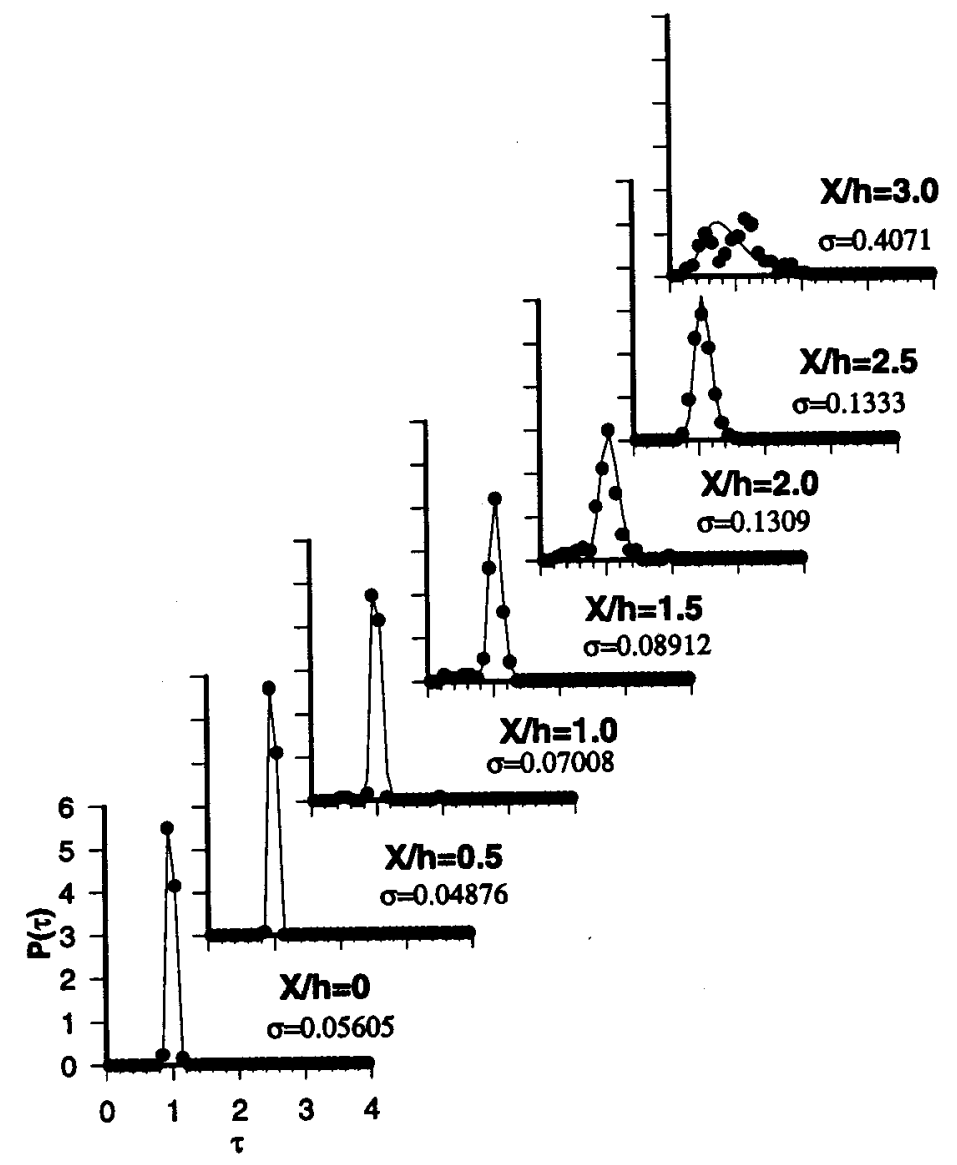

Figure 11. The phase jitter development of a forced reattaching shear layer forced at its natural frequency $=52 \mathrm{~Hz}$, along $U / U_{\max } \simeq 0.7$ using modified HZFB method.

are reported at three $X / h$ locations; $X / h=1.5$ representing the near field region, $X / h$ $=4.0$ representing the near-reattachment region and $X / h=9.0$ representing the postreattachment region. Upstream of reattachment, the measurements are reported only in the upper half of the shear layer due to the limitation of the $X$-wire in the presence of reversed flow. Different turbulent motions, i.e. ejection, sweep, outward interaction and wallward interaction, representing four quadrants in the $u^{\prime} v^{\prime}$ plane can be further partitioned based on the strengths of the eddies. Eddies of certain strength characterized by the hole size $H_{s}$ can be delineated by computing their contribution to $\overline{u^{\prime} v^{\prime}}$ only if the signal $\left|u^{\prime} v^{\prime}\right|$ is greater than the value $H_{s}\left|u^{\prime} v^{\prime}\right|_{r e f}$, where, $\left|u^{\prime} v^{\prime}\right|_{\text {ref }}$ represents the maximum absolute shear stress at all locations considered here $(X / h=1.5,4.0$ and 9.0). It should be noted that $\left|u^{\prime} v^{\prime}\right|_{\text {ref }}$ is generally taken to be equal to the total Reynolds stress at a streamwise location, while the maximum Reynolds stress for all $X / h$ and $Y / h$ locations is chosen here to allow consistent comparisons of eddy size between different locations. The contribution from different quadrants are calculated as a function of hole size, which provides the different eddy size in each quadrant. For a consistent comparison of eddy size between different locations and different quadrant motions the 


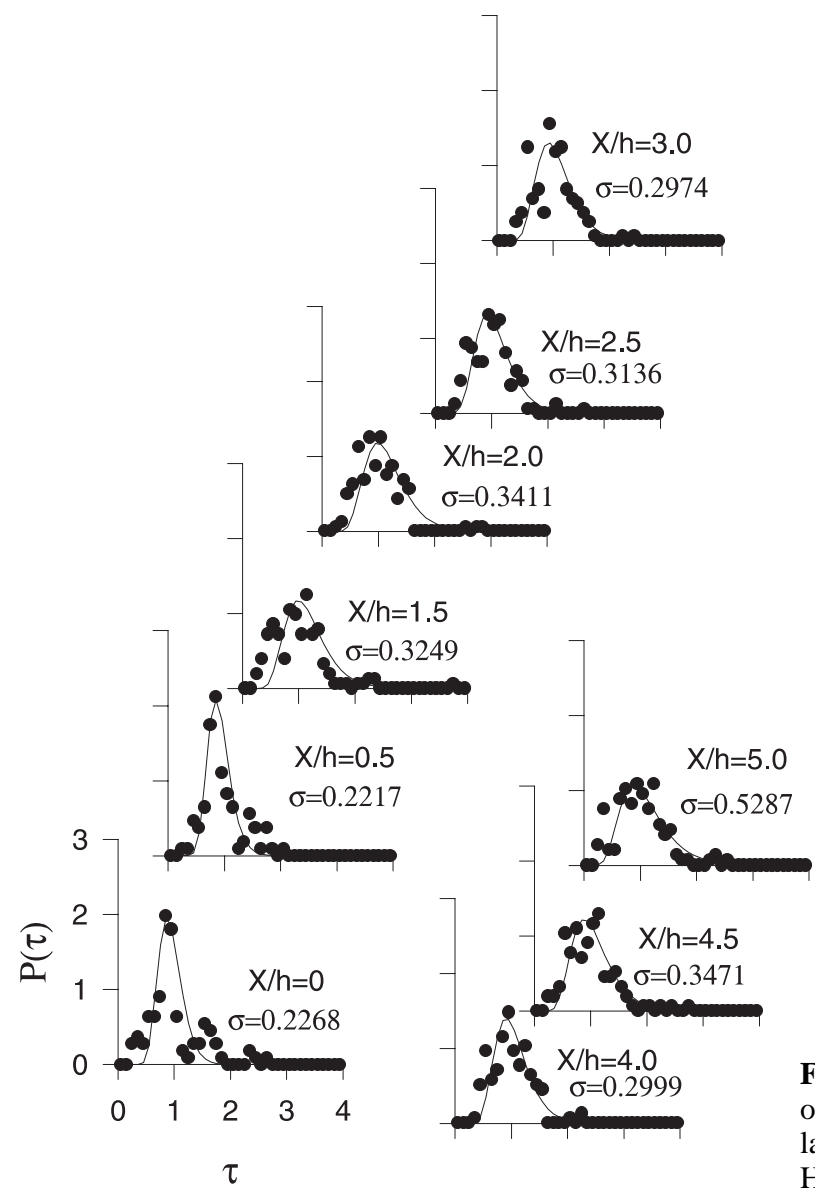

Figure 12. The phase jitter development of the naturally evolving reattaching shear layer along $U / U_{\max } \simeq 0.7$ using modified HZFB method.

average hole size is used. The average hole size is defined as,

$$
H_{s(\mathrm{Av})}=\int_{0}^{\infty}\left(u^{\prime} v^{\prime}\right) \mathrm{d} H_{s} /\left(u^{\prime} v^{\prime}\right)_{H_{s}=0} .
$$

The average hole size profile for the unexcited case at $X / h=1.5,4.0$ and 9.0 are presented in figure 14. The maximum average hole size for all motions increases in the downstream direction. Before reattachment, the wallward interaction has the smallest contribution but after reattachment, the wallward interaction is observed to have a larger hole size in the outer shear layer region than that of the outward interaction motion. Among all the motions, the ejection motion contribution has the largest scale eddies. The average hole size profiles for the excited case at $X / h=1.5,4.0$ and 9.0 locations are shown in figure 15. Comparing with the unexcited case, the average hole size is observed to be higher at the $X / h=1.5$ location for the excited case than for the unexcited case indicating larger growth of the large scales. The maximum average hole size of the ejection motion at $X / h=9.0$ for the excited case is smaller than that of the unexcited case, due to earlier saturations of the large-scale motions for the excited case. Thus, from the above discussion, it is observed that ejection has the largest scale motion among 


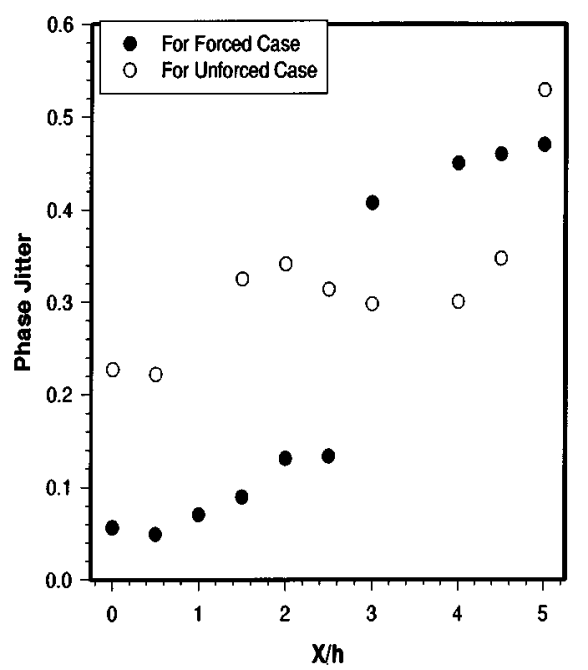

Figure 13. Comparison of phase jitter development in a reattaching shear layer for both forced and unforced cases at $U / U_{\max }=0.7$.

all quadrant motions and the maximum hole size for ejection motion is present at the outer region of the shear layer.

\section{Conclusions}

The behaviour of reattaching flow behind a rib mounted on the surface of a rectangular channel has been studied experimentally for both excited and unexcited cases. Different data analysis techniques for calculating the phase jitter and coherent structure magnitude of a turbulent signal is evaluated in this work using both simulated and reattaching shear layer data. For phase jitter calculation, the method used by Ho et al (1991) for mixing layers, referred to here as the HZFB method, is compared with the pattern recognition method and a modified HZFB method. For coherent structure magnitude calculation, the Fourier method and pattern recognition method are examined. The following observations are made.

(1) The modified HZFB technique and pattern recognition technique are observed to be successful in predicting the phase jitter evolution of a reattaching shear layer.

(2) The pattern recognition technique is successful in predicting the coherent structure magnitude of a turbulent signal, whereas the Fourier method and phase averaging method are erroneous in the presence of phase jitter in the signal.

(3) It is observed that the after-reattachment region is dominated by large-scale coherent structures contrary to the belief that during reattachment, large scales break into small scales, resulting in the predominant presence of small scales in the after-reattachment region.

(4) The results of this investigation do not support the conclusions from the numerical calculation of Arnal et al (1991), where it was noted that for a surface-mounted rib, vortices scale with the rib height $h$ and that the Strouhal number based on the rib height is a constant. The difference in behaviour may be attributed to the fact that Arnal et al (1991) assumed the flow to be laminar; while the flow is turbulent in nature. Instead, from this investigation, the correct length scale for Strouhal number calculation is observed to be the momentum thickness at the downstream rib corner. This Strouhal number is found to be about 0.013 , which is consistent with 0.012 value reported by Hasan (1992) for a backward-facing step. 

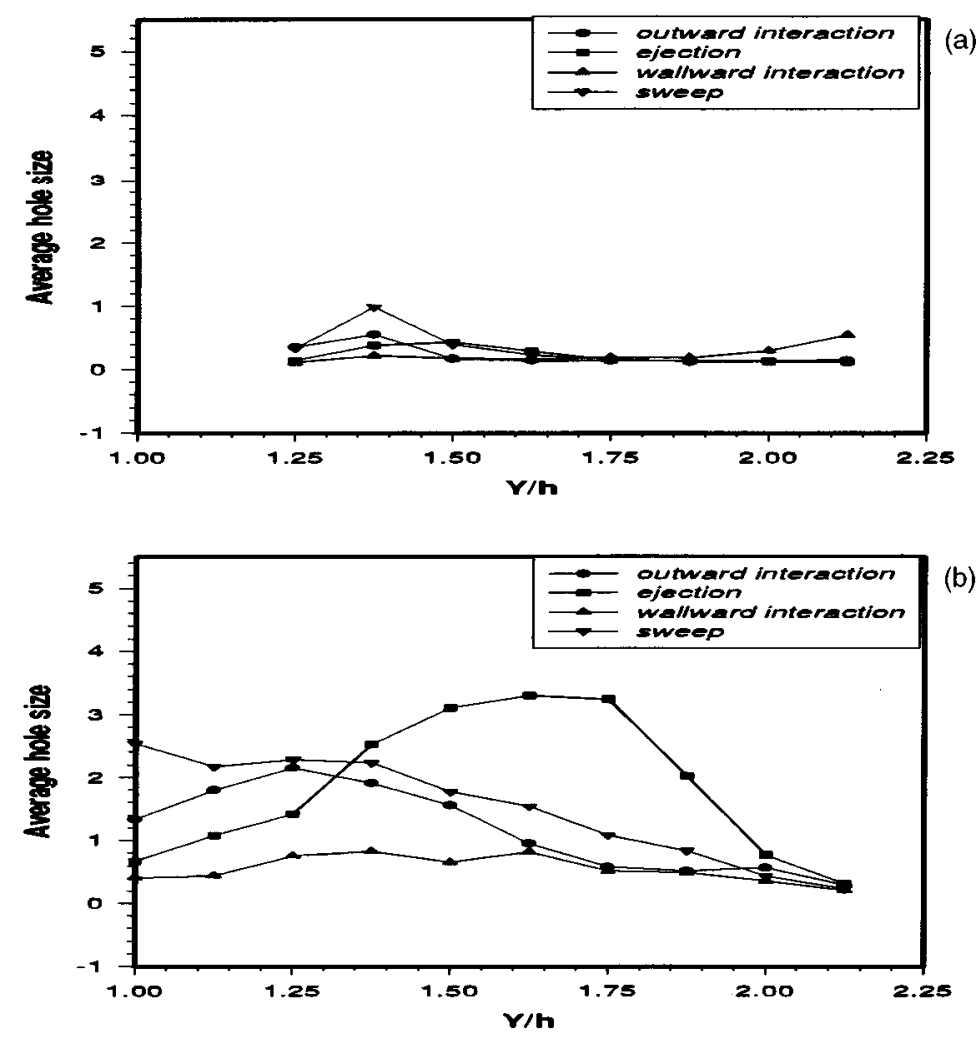

(b)

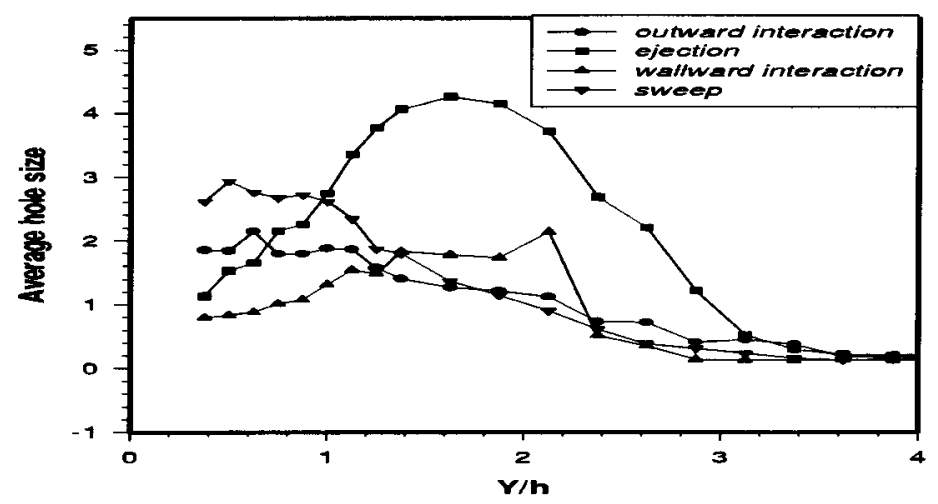

(c)

Figure 14. The average hole size profile of different quadrants at $X / h=1.5$ (a), 4.0 (b) and 9.0 (c) for the unexcited case.

(5) The phase jitter level of the reattaching shear layer is higher than that of the mixing layer in the near field region. The large-scale structures are well correlated in the near field region of the reattaching shear layer and the phase information is lost in the downstream direction at about $3 h$ from the downstream edge of the rib.

(6) Among all quadrant motions, the ejection motion is observed to be significant particularly in the outer region of the shear layer. 

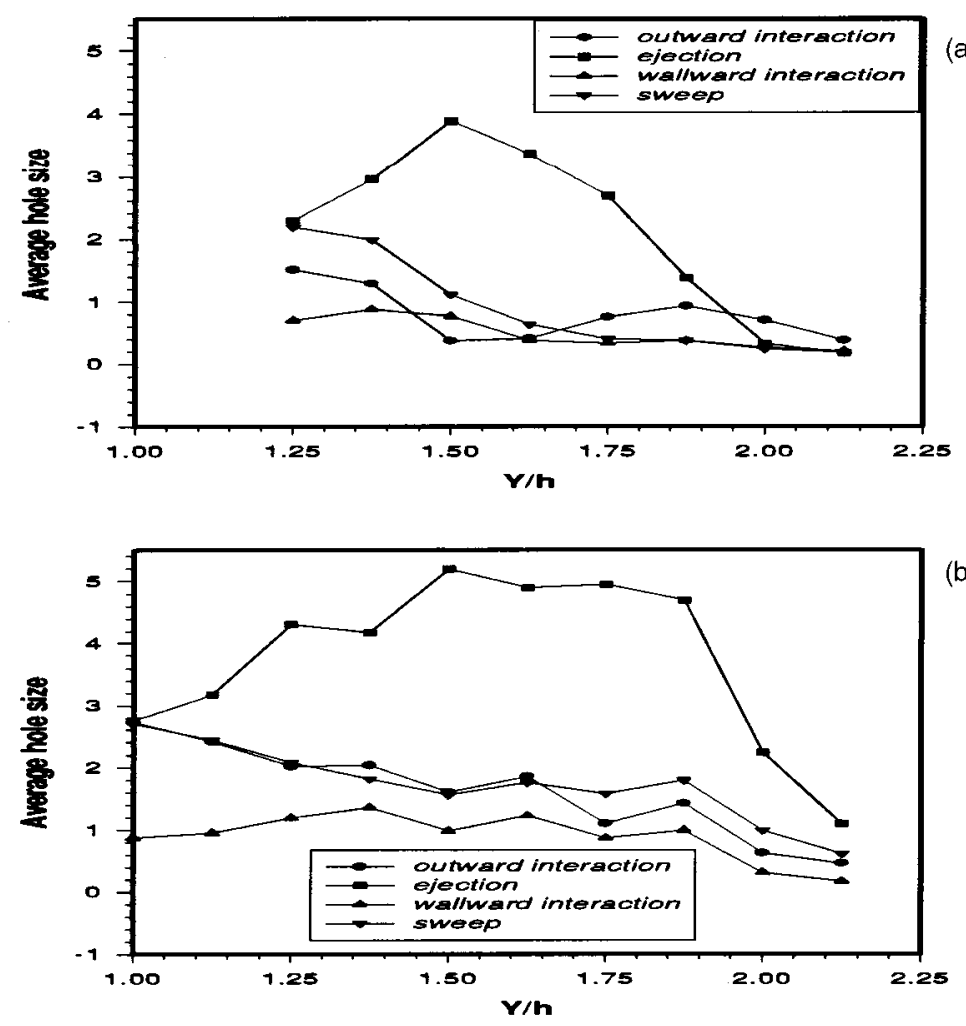

(b)

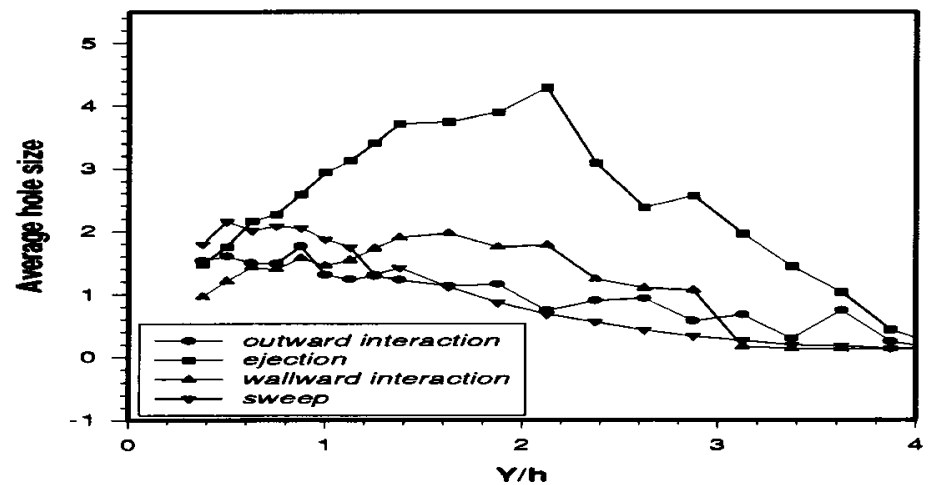

(c)

Figure 15. The average hole size profile of different quadrants at $X / h=1.5$ (a), 4.0 (b) and 9.0 (c) for the excited case.

\section{List of symbols}

A modal amplitude;

$(\mathrm{d} u / \mathrm{d} y)_{\max }$ maximum velocity gradient at the downstream rib corner;

$D_{h}$

hydraulic diameter of the channel $(2 W H /(W+H))=0.1013 \mathrm{~m}$;

$E(f) \quad$ power spectral density $\equiv(1 / T)\left(\left|(T / N) \sum_{n=1}^{N} U(n) \exp (-2 \pi i k n / N)\right|\right)^{2}$, $k=0,1,2 \ldots \ldots . . . N / 2$; 


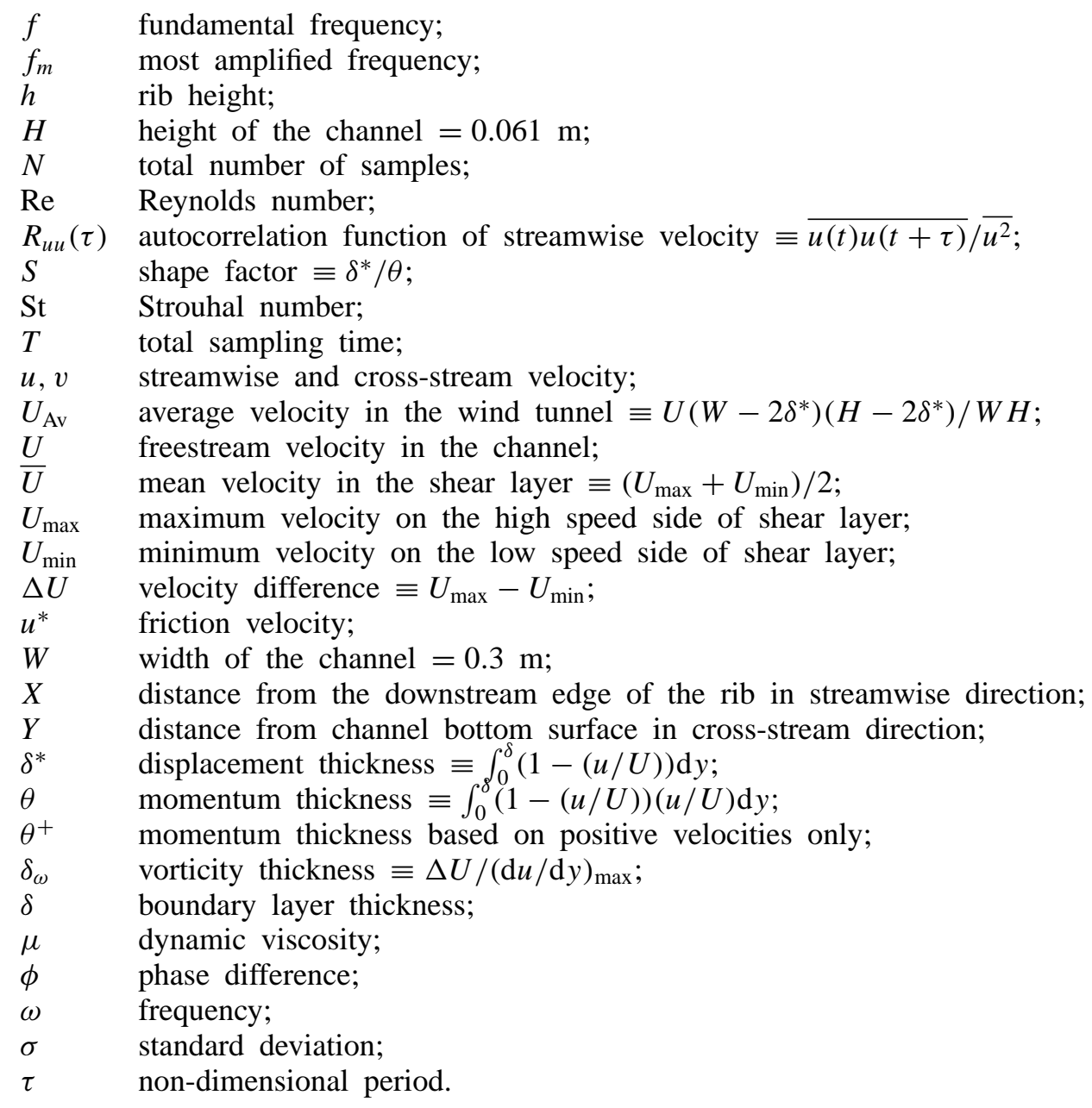

\section{Subscripts}

$c \quad$ coherent;

$r$ random;

$f \quad$ fundamental component;

$s \quad$ subharmonic component;

max maximum.

\section{References}

Arnal M P, Goering D J, Humphrey J A C 1991 Vortex shedding from a bluff body adjacent to a plane sliding wall. J. Fluid Eng. 113: 384-398

Fiedler H E, Mensing P 1985 The plane turbulent shear layer with periodic excitation. $J$. Fluid Mech. 150: 281-309

Hasan M A Z 1992 The flow over a backward facing step under controlled perturbation: Laminar separation. J. Fluid Mech. 238: 73-96 
Hinze J O 1975 Turbulence (New York: McGraw Hill)

Ho C M, Huere P 1984 Perturbed free shear layers. Annu. Rev. Fluid Mech. 16: 365-424

Ho C M, Zohar Y, Foss J K, Buell J C 1991 Phase decorrelation of coherent structures in a free shear layer. J. Fluid Mech. 230: 319-337

Huerre P, Monkewitz P A 1985 Absolute and convective instabilities in free shear layers. J. Fluid Mech. 159: 151-168

Hussain A K M F 1986 Coherent structures and turbulence. J. Fluid Mech. 173: 303-356

Hussain A K M F, Zaman K B M Q 1980 Vortex pairing in a circular jet under controlled excitation, J. Fluid Mech. 101: 493-544

Mcmanus K R, Vandsburger U, Bowman C T 1990 Combustor performance enhancement through direct shear layer excitation. Combust. Flame 82: 75-92

Najjar F M, Vanka S P 1993 Numerical study of a separated-reattaching flow. Theor. Comput. Fluid Dyn. 5: 291-308

Panigrahi PK, Acharya S 1996 Spectral characteristics of separated flow behind a surface-mounted square rib. 27th AIAA Fluid Dynamics Conference, AIAA 96-1931

Panigrahi P K, Acharya S 1999 Mechanisms of turbulence transport in a turbine blade coolant passage with a rib turbulator. J. Turbomachinery 121: 152-159

Rajaee M, Karlsson S K F 1992 On the Fourier space decomposition of free shear flow measurements and mode generation in the pairing process. Phys. Fluids A4: 321-339

Tam C K W 1978 Excitation of instability waves in a two-dimensional shear layer by sound. J. Fluid Mech. 89: 357-371

Wallace J M, Eckelmann H, Brodkey R S 1972 The wall region in turbulent shear flow. J. Fluid Mech. 54: 39-48

Willmarth W W, Lu S S 1972 Structure of the Reynolds stress near the wall. J. Fluid Mech. 55: 65-92

Yang Z, Karlsson S K F 1991 Evolution of coherent structures in a plane shear layer. Phys. Fluids A3: 2207-2219

Zhou M D, Heine C, Wygnanski I 1996 The effects of excitation on the coherent and random motion in a plane wall jet. J. Fluid Mech. 310: 1-37 